\title{
GLOBAL MIXED PERIODS AND LOCAL KLYACHKO MODELS FOR THE GENERAL LINEAR GROUP
}

\author{
OMER OFFEN AND EITAN SAYAG
}

\begin{abstract}
We show that every irreducible representation in the discrete automorphic spectrum of $G L_{n}(\mathbb{A})$ admits a non vanishing mixed (Whittaker-symplectic) period integral. The analog local problem is a study of models first considered by Klyachko over a finite field. Locally, we show that for a $p$-adic field $F$ every irreducible, unitary representation of $G L_{n}(F)$ has a Klyachko model.
\end{abstract}

\section{INTRODUCTION}

Fundamental to the theory of automorphic forms on $G L_{n}$ is the fact that a cuspidal automorphic representation admits a global Whittaker functional. Other period integrals were considered for certain representations in the residual spectrum. The study of global symplectic period integrals for $G L_{n}$ was initiated by Jacquet and Rallis in [JR92]. They were further studied in Off06a]. In Off06b, the first named author characterized all irreducible representations in the discrete automorphic spectrum that admit a symplectic period. The main global result of the present work provides a non zero period integral for any irreducible representation in the discrete spectrum. Namely, following Heu93, we consider a certain finite list of Whittaker-symplectic period integrals and show that every discrete spectrum representation of $G L_{n}$ admits one of them.

The mixed period integral is factorizable (see Corollary 1) and our global results have local analogues. The local results of this work continue the study of symplectic models considered in [HR90, OS07]. In K184, Klyachko introduced certain mixed (Whittaker-symplectic) models in the context of $G L_{n}$ over a finite field. Our main local result extends the work of Heumos and Rallis. We show that every irreducible, unitary representation of $G L_{n}$ over a $p$-adic field has a Klyachko model. This was previously obtained in [HR90] for $n \leq 4$. See also [N07].

To describe our results more precisely, we set the necessary notation. Let $F$ be either a number field or a $p$-adic field. In the global case, denote by $\mathbb{A}=\mathbb{A}_{F}$ the ring of adèles of $F$. Let $G_{r}=G L_{r}$ be regarded 
as an algebraic group defined over $F$ and let $U_{r}$ denote the group of upper triangular unipotent matrices in $G_{r}$.

Fix $n$ and let $G=G_{n}$. For any decomposition $n=r+2 k$ we consider a subgroup of $G_{n}$ defined by

$$
H_{r, 2 k}=\left\{\left(\begin{array}{cc}
u & X \\
0 & h
\end{array}\right) \in G: u \in U_{r}, X \in M_{r \times 2 k} \text { and } h \in S p(2 k)\right\} .
$$

Here

$$
S p(2 k)=\left\{g \in G_{2 k}:{ }^{t} g\left(\begin{array}{ll}
w_{k} \\
-w_{k}
\end{array}\right) g=\left(\begin{array}{cc}
w_{k} \\
-w_{k}
\end{array}\right)\right\}
$$

and $w_{k} \in G_{k}$ is the permutation matrix whose $(i, j)$ th entry is $\delta_{k+1-i, j}$. Let $\psi$ be a non trivial character of $F$ in the local case (resp. of $F \backslash \mathbb{A}$ in the global case). We associate to $\psi$ the character $\psi_{r}$ of $U_{r}(F)$ (resp. of $\left.U_{r}(F) \backslash U_{r}(\mathbb{A})\right)$ defined by

$$
\psi_{r}(u)=\psi\left(u_{1,2}+\cdots+u_{r-1, r}\right) .
$$

By abuse of notation we will also denote by $\psi_{r}$ the character of $H_{r, 2 k}(F)$ (resp. of $H_{r, 2 k}(F) \backslash H_{r, 2 k}(\mathbb{A})$ ) defined by

$$
\psi_{r}\left(\begin{array}{cc}
u & X \\
0 & h
\end{array}\right)=\psi_{r}(u)
$$

We now describe our main results, first in the global case and then in the local case.

1.1. The Global case. Let $F$ be a number field with adèle ring $\mathbb{A}$. We denote by $Z_{G}$ the center of $G$. Fix once and for all a unitary character $\xi$ of $Z_{G}(F) \backslash Z_{G}(\mathbb{A})$ and denote by $L^{2}\left(Z_{G}(\mathbb{A}) G(F) \backslash G(\mathbb{A}), \xi\right)$ the space of functions $\phi$ on $G(F) \backslash G(\mathbb{A})$ such that $\phi(z g)=\xi(z) \phi(g)$ for all $z \in Z_{G}(\mathbb{A}), g \in G(\mathbb{A})$ and

$$
\int_{Z_{G}(\mathbb{A}) G(F) \backslash G(\mathbb{A})}|\phi(g)|^{2} d g<\infty .
$$

We have an orthogonal decomposition

$$
L^{2}\left(Z_{G}(\mathbb{A}) G(F) \backslash G(\mathbb{A}), \xi\right)=L_{\text {disc }}^{2}(G, \xi) \oplus L_{\text {cont }}^{2}(G, \xi)
$$

of the automorphic spectrum into a discrete part and a continuous part. The discrete part decomposes further as a direct sum of irreducible representations, each appearing with multiplicity one. We say that $\pi$ is a discrete spectrum automorphic representation of $G(\mathbb{A})$ with central character $\xi$ if it embeds into $L_{\text {disc }}^{2}(G, \xi)$ and we refer to this embedding as the automorphic realization of $\pi$. In [MW89, Møglin and Waldspurger show that the irreducible components of $L_{d i s c}^{2}(G, \xi)$ are precisely the representations $L(\sigma, t)$ parameterized by pairs $(\sigma, t)$ 
where $n=r t$ and $\sigma$ is a cuspidal automorphic representation of $G_{r}(\mathbb{A})$ with central character appropriately related to $\xi$ (see the first paragraph of $\$ 2$ for notation and the precise statement).

Let $H$ be an algebraic subgroup of $G$ and let $\chi$ be a character of $H(F) \backslash H(\mathbb{A})$. For an automorphic form $\phi$, whenever the integral converges, we define

$$
l_{H}^{\chi}(\phi)=\int_{H(F) \backslash H(\mathbb{A})} \phi(h) \chi(h) d h .
$$

When $\chi$ is the trivial character we will also write $l_{H}$ for $l_{H}^{\chi}$.

Definition 1. We say that an automorphic representation $\pi$ is $(H, \chi)-$ distinguished if there is an automorphic form $\phi$ in the space of $\pi$ such that $l_{H}^{\chi}(\phi) \neq 0$. When $\chi$ is the trivial character we will then say that $\pi$ is $H$-distinguished.

Our main global result is expressed in terms of the classification of the discrete spectrum as follows.

Theorem 1. Let $\pi$ be an irreducible, discrete spectrum automorphic representation of $G(\mathbb{A})$ (of central character $\xi$ ). Then, there exists an integer $k, 0 \leq k \leq\left[\frac{n}{2}\right]$ such that $\pi$ is $\left(H_{n-2 k, 2 k}, \psi_{n-2 k}\right)$-distinguished. More precisely, if $\pi=L(\sigma, t)$ and

$$
\kappa(\pi)=r\left[\frac{t}{2}\right]
$$

then $\pi$ is $\left(H_{n-2 \kappa(\pi), 2 \kappa(\pi)}, \psi_{n-2 \kappa(\pi)}\right)$-distinguished.

Discrete spectrum automorphic representations are realized as multiresidues of Eisenstein series. Our proof of Theorem 4 (that also implies Theorem 11) relies on formula (8) that expresses the mixed period $l_{H_{n-2 k, 2 k}}^{\psi_{n-2 k}}$ of the multi-residue of an Eisenstein series in terms of Whittaker and purely symplectic periods.

1.2. The local case. Let $F$ be a $p$-adic field. We will consider only smooth representations of $G(F)$. In particular, when we say that the representation $\pi$ of $G(F)$ is unitary we really mean that $\pi$ is a smooth representation that has a unitary structure.

Let $H$ be an algebraic subgroup of $G$ and let $\chi$ be a character of $H(F)$.

Definition 2. We say that a representation $\pi$ of $G(F)$ is $(H, \chi)-$ distinguished if $\operatorname{Hom}_{H(F)}(\pi, \chi) \neq 0$. If $\chi$ is the trivial character we also say that $\pi$ is $H$-distinguished. 
Theorem 2. Let $\pi$ be an irreducible, unitary representation of $G(F)$. There exists an integer $k, 0 \leq k \leq\left[\frac{n}{2}\right]$ such that $\pi$ is $\left(H_{n-2 k, 2 k}, \psi_{n-2 k}\right)-$ distinguished.

As in the global case, following our main local result, Theorem 8 , we construct a map $\pi \mapsto \kappa(\pi)$ that assigns (in particular) to any irreducible, unitary representation an integer $k=\kappa(\pi)$ for which Theorem 2 holds. This map is described explicitly in \$3 in terms of Tadic's classification of the unitary dual of $G(F)$ obtained in [Tad86]. Our proof is local. It is based, however, on the hereditary property of Whittaker models with respect to parabolic induction and on our results on purely symplectic models in OS07. We remark that our proof in OS07] uses a global argument. Thus, our entire proof is based on the global theory of automorphic forms. It will be interesting to see a purely local proof of Theorem 2 .

1.3. Some background on the study of mixed periods. Theorem 2 can be interpreted as an existence statement of certain mixed models. For a decomposition $n=r+2 k$ we introduce the Klyachko model

$$
\mathcal{M}_{r, 2 k}=\operatorname{Ind}_{H_{r, 2 k}(F)}^{G(F)}\left(\psi_{r}\right) \text {. }
$$

Note that $\mathcal{M}_{n, 0}$ is the Whittaker model while when $n$ is even $\mathcal{M}_{0, n}$ is a purely symplectic model. By Frobenious reciprocity, for any admissible representation $\pi$ of $G(F)$ we have

$$
\operatorname{Hom}_{G(F)}\left(\pi, \mathcal{M}_{r, 2 k}\right)=\operatorname{Hom}_{H_{r, 2 k}(F)}\left(\pi, \psi_{r}\right) .
$$

Thus, if $\pi$ is irreducible then it is $\left(H_{r, 2 k}, \psi_{r}\right)$-distinguished if and only if it can be realized in the space $\mathcal{M}_{r, 2 k}$. The Klyachko model $\mathcal{M}_{r, 2 k}$ is a mixed (Whittaker-symplectic) model for $G(F)$ and whenever $\pi$ is $\left(H_{r, 2 k}, \psi_{r}\right)$-distinguished we say that $\pi$ admits the model $\mathcal{M}_{r, 2 k}$.

The models $\mathcal{M}_{r, 2 k}$ were first considered by Klyachko in [Kl84] in the case where $F$ is a finite field. If $F=\mathbb{F}_{q}$ is the field of $q$ elements, the work of Klyachko suggests that

$$
\mathcal{M}=\oplus_{k=0}^{\left[\frac{n}{2}\right]} \mathcal{M}_{n-2 k, 2 k}
$$

is a Gelfand model for $G\left(\mathbb{F}_{q}\right)$, i.e. it is the direct sum of all irreducible representations of $G\left(\mathbb{F}_{q}\right)$ each appearing with multiplicity one. In other words, for any irreducible representation $\pi$ of $G\left(\mathbb{F}_{q}\right)$ we have $m_{\pi}=1$ where $m_{\pi}$ is defined by

$$
m_{\pi}=\operatorname{dim}_{\mathbb{C}}\left(\operatorname{Hom}_{G(F)}(\pi, \mathcal{M})\right) .
$$

As already pointed out by Inglis and Saxl the proof of Klyachko contains several inaccuracies and gaps and is therefore incomplete. In 
[S91 a complete proof is given, using different methods. The result has applications to the representation theory of $G\left(\mathbb{F}_{q}\right)$ and was used for example in [FG03] and in Vin06. In TV05, an analog is proved for the finite unitary group.

The fact that $m_{\pi}=1$ for an irreducible representation $\pi$ consists of the following 3 properties: existence ( $\pi$ admits some Klyachko model), disjointness ( $\pi$ admits at most one Klyachko model) and uniqueness ( $\pi$ imbeds into a given Klyachko model with at most multiplicity one). Klyachko models over a $p$-adic field were first studied by Heumos and Rallis in HR90. They observed that, already when $n=3$ there exists an irreducible, admissible representation $\pi$ of $G(F)$ that admits no Klyachko model, i.e. such that $m_{\pi}=0$. However, when $n \leq 4$ they showed that every irreducible, unitary representation $\pi$ of $G(F)$ admits a Klyachko model, i.e. that $m_{\pi} \geq 1$. In general, they also showed the uniqueness of the purely symplectic model [HR90, Theorem 2.4.2], i.e. that if $n$ is even and $\pi$ is an irreducible admissible representation of $G(F)$ then

$$
\operatorname{dim}_{\mathbb{C}}\left(\operatorname{Hom}_{G(F)}\left(\pi, \mathcal{M}_{0, n}\right)\right) \leq 1 .
$$

Another result claimed in [HR90, Theorem 3.1] is disjointness of Klyachko models for irreducible, unitary representations. Unfortunately, the proof is based on [K184, Proposition 1.3] which is false. To be more precise, the proof given in [HR90, §3] could have been based on the statement in [K184, §1.1], which is a weaker statement then [K184, Proposition 1.3] and which may still be true but, to our knowledge, has not yet been proved. We will obtain the disjointness and uniquness of Klyachko models over a $p$-adic field in an upcoming paper. The local part of the current paper treats the existence of Klyachko models.

In [OS07, we provided a family of unitary representations of $G(F)$ that admit a purely symplectic model. From Theorem 2 we get that $m_{\pi} \geq 1$ for any irreducible unitary representation $\pi$ of $G(F)$. We also promised in [OS07] that the current work will characterize, in particular, all irreducible unitary representations admitting a symplectic model. This was based on the unitary disjointness that, we only later observed, remains unproved. We will therefore only deliver our promise in our upcoming paper when we prove disjointness of the Klyachko models. It will also be interesting to study the analogous problem in the archimedean case, and the global mixed periods for the continuous automorphic spectrum of $G$. Langlands, described the continuous spectrum in terms of discrete spectrum datum. Using this description, we believe that a global analogue of Theorem 2, properly formulated, should be true and we also hope to address this problem in the future. 
Our study of the global mixed periods was motivated by its analogy with the local problem. This analogy, was already suggested by Heumos in his survey paper on the subject [Heu93].

Remark 1. The focus of this paper is on non vanishing of periods. Thus, the way Haar measures are normalized plays no role in the proofs of the main results. We therefore do not choose a specific normalization for the Haar measures appearing in this work and (with the exception of \$2.3) will make no further comments regarding the choice of measures.

1.4. Acknowledgement. We are grateful to Joseph Bernstein for his help and patience. His insistence for conceptual arguments significantly simplified this work.

\section{GLOBAL MIXED PERIODS}

Mœglin and Waldspurger classified the discrete spectrum automorphic representations of $G(\mathbb{A})$ in [MW89]. We recall their result.

Let $P=M U$ be a standard parabolic subgroup of $G$ with Levi subgroup $M$ and unipotent radical $U$. If $P$ is of type $\left(n_{1}, \ldots, n_{t}\right)$ then for an automorphic representation $\tau=\tau_{1} \otimes \cdots \otimes \tau_{t}$ of $M(\mathbb{A})$ and for $\lambda=\left(\lambda_{1}, \ldots, \lambda_{t}\right) \in \mathbb{C}^{t}$ let $\tau[\lambda]$ be the representation on the space of $\tau$ defined by

$$
\tau[\lambda]=|\operatorname{det}|^{\lambda_{1}} \tau_{1} \otimes \cdots \otimes|\operatorname{det}|^{\lambda_{t}} \tau_{t}
$$

and let $I(\tau, \lambda)=I_{P}^{G}(\tau, \lambda)$ be the representation of $G(\mathbb{A})$ parabolically induced from $\tau[\lambda]$. For a positive integer $t$ let

$$
\Lambda_{t}=\left(\frac{t-1}{2}, \frac{t-3}{2}, \ldots, \frac{1-t}{2}\right) .
$$

Fix a character $\xi$ of $F^{\times} \backslash \mathbb{A}^{\times}$and identify $Z_{G}(\mathbb{A})$ with $\mathbb{A}^{\times}$. Let $r t=n$ and let $\sigma$ be an irreducible, cuspidal automorphic representation of $G_{r}(\mathbb{A})$. Assume that $P$ is of type $(r, \ldots, r)$ and let $\tau=\sigma^{\otimes t}$. The representation $I\left(\tau, \Lambda_{t}\right)$ has a unique irreducible quotient, which we denote by $L(\sigma, t)$. Note that the central character of $I\left(\tau, \Lambda_{t}\right)$ and therefore also of $L(\sigma, t)$ is $\omega_{\sigma}^{t}$ where $\omega_{\sigma}$ is the central character of $\sigma$.

Theorem 3 (Møglin-Waldspurger). Let $n=r t$ and let $\sigma$ be an irreducible, cuspidal automorphic representation of $G_{r}(\mathbb{A})$ so that $\omega_{\sigma}^{t}=\xi$. The representation $L(\sigma, t)$ occurs in $L_{\text {disc }}^{2}(G, \xi)$ with multiplicity one and every irreducible component of $L_{\text {disc }}^{2}(G, \xi)$ is of the form $L(\sigma, t)$ for such a pair $(\sigma, t)$.

We now turn to the proof of Theorem 1.1. Let $\pi=L(\sigma, t)$ be an irreducible component of $L_{\text {disc }}^{2}(G, \xi)$. If $\pi$ is cuspidal (i.e. if $t=1$ ), it is well known that the Whittaker functional $l_{U_{n}}^{\psi_{n}}$ is not identically zero 
on $\pi$, in other words $\pi$ is $\left(U_{n}, \psi_{n}\right)$-distinguished. On the other hand, in [Off06b, Theorem 3] we show that $\pi$ is $S p(n)$-distinguished if and only if $t$ is even. Theorem 1 therefore follows from

Theorem 4. Let $n=(2 m+1) r$ and let $\sigma$ be an irreducible, cuspidal automorphic representation of $G_{r}(\mathbb{A})$. The representation $L(\sigma, 2 m+1)$ is $\left(H_{r, 2 m r}, \psi_{r}\right)$-distinguished.

The proof of Theorem 4 is given in $₫ 2.2$. We start with some notation and a summary of necessary facts.

\subsection{Eisenstein series, intertwining operators and multi-residues.} When we say that $P=M U$ is a standard parabolic subgroup of $G$ with its standard Levi decomposition, we mean that $M$ is the standard Levi subgroup and $U$ is the unipotent radical of $P$. Throughout, $P=M U$ and $Q=L V$ will denote standard parabolic subgroups of $G$ with their standard Levi decompositions so that $P$ is contained in $Q$.

For integers $a \leq b$ let $[a, b]=\{a, a+1, \ldots, b\}$. We identify the index set $\Delta=[1, n-1]$ with the set of simple roots of $G$ with respect to the standard Borel subgroup of upper triangular matrices in $G$ and let $\Delta^{M}$ denote the set of those indices $i$ that correspond to roots in $M$. If $P$ is of type $\left(n_{1}, \ldots, n_{t}\right)$ then

$$
\Delta^{M}=\Delta \backslash\left\{n_{1}, n_{1}+n_{2}, \ldots, n_{1}+\cdots+n_{t-1}\right\} .
$$

Let $S_{n}$ denote the group of permutations on $[1, n]$. We denote by $W_{M}$ the Weyl group of $M$ and let $W=W_{G}$. We identify $W$ with $S_{n}$ and when convenient we consider an element of $S_{n}$ as a permutation matrix in $G$. Each double coset in $W_{L} \backslash W / W_{M}$ contains a unique representative of minimal length - the left $W_{L}$ and right $W_{M}$ reduced representative. We denote by ${ }_{L} W_{M}$ the set of reduced representatives of the double cosets and let

$$
{ }_{L} W_{M}^{c}=\left\{w \in{ }_{L} W_{M}: w M w^{-1} \subset L\right\} .
$$

If $n=t r$ and $P$ is of type $(r, \ldots, r)$ the set ${ }_{M} W_{M}^{c}$ consists of the Weyl elements permuting the blocks of $M$ and we identify ${ }_{M} W_{M}^{c}$ with $S_{t}$.

For an algebraic group $Y$ defined over $F$ let $X^{*}(Y)$ be the lattice of rational characters of $Y$, let $\mathfrak{a}_{Y}^{*}=X^{*}(Y) \otimes \mathbb{R}$ and let $\mathfrak{a}_{Y}$ be the dual vector space. Denote by $\langle\cdot, \cdot\rangle$ the pairing between $\mathfrak{a}_{Y}$ and $\mathfrak{a}_{Y}^{*}$. If $P$ is of type $\left(n_{1}, \ldots, n_{t}\right)$ then we identify $\mathfrak{a}_{M}$ and its dual with $\mathbb{R}^{t}$. The pairing between $\mathfrak{a}_{M}$ and $\mathfrak{a}_{M}^{*}$ is then the standard inner product on $\mathbb{R}^{t}$. There is a natural embedding of $\mathfrak{a}_{L}$ into $\mathfrak{a}_{M}$ with an orthogonal decomposition

$$
\mathfrak{a}_{M}=\mathfrak{a}_{L} \oplus \mathfrak{a}_{M}^{L} .
$$


Denote by $X \mapsto X_{L}$ the orthogonal projection from $\mathfrak{a}_{M}$ to $\mathfrak{a}_{L}$. We use similar notation for the corresponding decomposition in the dual space and denote by $\lambda \mapsto \lambda_{L}$ the orthogonal projection of $\mathfrak{a}_{M}^{*}$ to $\mathfrak{a}_{L}^{*}$. Using the Iwasawa decomposition $G(\mathbb{A})=U(\mathbb{A}) M(\mathbb{A}) K$, where $K$ is the standard maximal compact subgroup of $G(\mathbb{A})$, we define the height function $H_{M}: G(\mathbb{A}) \rightarrow \mathfrak{a}_{M}$ by the requirement that for every $\chi \in X^{*}(M)$ we have

$$
e^{\langle\chi, H(u m k)\rangle}=\prod_{v}\left|\chi_{v}\left(m_{v}\right)\right|_{v} .
$$

The map $H_{M}$ induces an isomorphism $M(\mathbb{A}) / M(\mathbb{A})^{1} \rightarrow \mathfrak{a}_{M}$. Let $\rho_{P}=$ $\left(\Lambda_{n}\right)_{M} \in\left(\mathfrak{a}_{M}\right)^{*}$ where $\Lambda_{n}$ is given by (3). Thus,

$$
p \mapsto e^{\left\langle 2 \rho_{P}, H_{M}(p)\right\rangle}
$$

is the modulus function of $P(\mathbb{A})$.

Let $\tau$ be an irreducible, cuspidal automorphic representation of $M(\mathbb{A})$. We identify the representation spaces of $I_{P}^{G}(\tau, \lambda)$ for all $\lambda \in \mathfrak{a}_{M \text {, } \mathbb{C}}^{*}$ with the representation space $I_{P}^{G}(\tau)$ with $\lambda=0$. In particular, we have

$$
\varphi(p g)=e^{\left\langle\rho_{P}, H_{M}(p)\right\rangle} \tau(m) \varphi(g)
$$

whenever $\varphi \in I_{P}^{G}(\tau), p=m u \in P(\mathbb{A}), m \in M(\mathbb{A}), u \in U(\mathbb{A})$ and $g \in G(\mathbb{A})$. For $\varphi \in I_{P}^{G}(\tau)$ and $\lambda \in \mathfrak{a}_{M, \mathbb{C}}^{*}$ we denote by $\varphi_{\lambda}$ the standard holomorphic section given by

$$
\varphi_{\lambda}(g)=\varphi(g) e^{\left\langle\lambda, H_{M}(g)\right\rangle} .
$$

The action of the representation $I_{P}^{G}(\tau, \lambda)$ on the space $I_{P}^{G}(\tau)$ is then given by

$$
\left(I_{P}^{G}(g, \tau, \lambda) \varphi\right)_{\lambda}(x)=\varphi_{\lambda}(x g)
$$

for $g, x \in G(\mathbb{A})$.

Let $\lambda \in \mathfrak{a}_{M, \mathbb{C}}^{*}$ and let $\varphi \in I_{P}^{G}(\tau)$. The Eisenstein series $E^{Q}(\varphi, \lambda)$ is defined as the meromorphic continuation of the series

$$
E^{Q}(g, \varphi, \lambda)=\sum_{\gamma \in(P \cap L)(F) \backslash L(F)} \varphi_{\lambda}(\gamma g) .
$$

When $Q=G$ we also set $E(\varphi, \lambda)=E^{Q}(\varphi, \lambda)$.

Assume now that $n=t r$, that $P$ is of type $(r, \ldots, r)$, that $Q$ is of type $\left(m_{1} r, \ldots, m_{s} r\right)$, that $\sigma$ is an irreducible, cuspidal automorphic representation of $G_{r}(\mathbb{A})$ and that $\tau=\sigma^{\otimes t}$ is the corresponding cuspidal representation of $M(\mathbb{A})$. For any $w \in{ }_{M} W_{M}^{c}=S_{t}$ we let $M(w, \lambda): I(\tau, \lambda) \rightarrow I(\tau, w \lambda)$ be the standard (non normalized) intertwining operator. It is defined by the meromorphic continuation of the 
integral

$$
(M(w, \lambda) \varphi)_{w \lambda}(g)=\int_{\left(U \cap w U w^{-1}\right)(\mathbb{A}) \backslash U(\mathbb{A})} \varphi_{\lambda}\left(w^{-1} u g\right) d u .
$$

Its domain of convergence includes that of the Eisenstein series and it admits a meromorphic continuation. Let

$$
\Lambda^{Q}=\left(\Lambda_{m_{1}}, \ldots, \Lambda_{m_{s}}\right) \in\left(\mathfrak{a}_{M}^{L}\right)^{*}
$$

and let $\mu \in \mathfrak{a}_{L}^{*}$. For $\varphi \in I_{P}^{G}(\tau)$ the expression

$$
E^{Q}(\varphi, \lambda+\mu) \prod_{i \in \Delta^{L}}\left(\lambda_{i}-\lambda_{i+1}-1\right)
$$

is holomorphic at $\lambda=\Lambda^{Q}$. We may therefore define the multi-residue of the Eisenstein series by

$$
E_{-1}^{Q}(\varphi, \mu)=\lim _{\lambda \rightarrow \Lambda^{Q}} E^{L}(\varphi, \lambda+\mu) \prod_{i \in \Delta^{L}}\left(\lambda_{i}-\lambda_{i+1}-1\right) .
$$

It defines a surjective intertwining operator

$$
E_{-1}^{Q}(\mu): I_{P}^{G}\left(\tau, \Lambda^{Q}+\mu\right) \rightarrow I_{Q}^{G}\left(L\left(\sigma, m_{1}\right) \otimes \cdots \otimes L\left(\sigma, m_{t}\right), \mu\right) .
$$

When $Q=G$ we also denote the multi-residue operator by $E_{-1}=$ $E_{-1}^{G}(0)$. It is then a surjective intertwining operator from $I\left(\tau, \Lambda_{t}\right)$ to $L(\sigma, t)$ that realizes the representation $L(\sigma, t)$ in the space $L_{d i s c}^{2}(G, \xi)$ of automorphic forms. We also consider the multi-residue of the intertwining operator $M(w, \lambda)$. Let

$$
\Delta(w)=\{i \in[1, t-1]: w(i)>w(i+1)\}
$$

and set

$$
M_{-1}(w)=\lim _{\lambda \rightarrow \Lambda_{t}} M(w, \lambda) \prod_{i \in \Delta(w)}\left(\lambda_{i}-\lambda_{i+1}+1\right) .
$$

It is an intertwining operator from $I\left(\tau, \Lambda_{t}\right)$ to $I\left(\tau, w \Lambda_{t}\right)$. For an automorphic form $\phi$ on $G(\mathbb{A})$ we define its constant term along $Q$ by

$$
\phi_{Q}(g)=\int_{V(F) \backslash V(\mathbb{A})} \phi(v g) d v .
$$

The function $\ell \mapsto \phi_{Q}(\ell)$ is an automorphic form on $L(\mathbb{A})$. We denote it by $\phi_{Q}[e]$. 
2.2. Proof of Theorem 4. Fix a decomposition $n=(2 m+1) r$. Let $H=H_{r, 2 m r}$, let $P$ be of type $(r, \ldots, r)$ and let $Q=L V$ be of type $(r, 2 m r)$ (i.e. $Q$ is the standard maximal parabolic subgroup of $G$ containing $H)$. Note that

$$
H \simeq\left(U_{r} \times S p(2 m r)\right) V \text { and } L \simeq G_{r} \times G_{2 m r} .
$$

If $\phi$ is an automorphic form in the discrete spectrum then it is easy to see that

$$
l_{H}^{\psi_{r}}(\phi)=\left(l_{U_{r}}^{\psi_{r}} \otimes l_{S p(2 m r)}\right)\left(\phi_{Q}[e]\right) .
$$

Let $\sigma$ be a cuspidal automorphic representation of $G_{r}(\mathbb{A})$, let $\tau=$ $\sigma^{\otimes 2 m+1}$ and let $\pi=L(\sigma, 2 m+1)$. Our goal is to show that the mixed period $l_{H}^{\psi_{r}}$ is not identically zero on $\pi$. As already explained, it is known that $l_{U_{r}}^{\psi_{r}} \otimes l_{S p(2 m r)}$ is not zero on $\sigma[-m] \otimes L(\sigma, 2 m)\left[\frac{1}{2}\right]$. From (6) we see that it is enough to show the following.

Proposition 1. The map $\phi \mapsto \phi_{Q}[e]$ defines a surjection from $\pi$ to $\sigma[-m] \otimes L(\sigma, 2 m)\left[\frac{1}{2}\right]$.

Proof. Note first that $\sigma[-m] \otimes L(\sigma, 2 m)\left[\frac{1}{2}\right]$ is irreducible and therefore it is enough to prove that the map $\phi \mapsto \phi_{Q}[e]$ is not identically zero on $\pi$ and that its image indeed lies in

$$
\sigma[-m] \otimes L(\sigma, 2 m)\left[\frac{1}{2}\right] .
$$

Since $\phi_{Q}(\ell g)$ is the value at $\ell$ of $(\pi(g) \phi)_{Q}[e]$ for $\ell \in L(\mathbb{A}), g \in G(\mathbb{A})$, to prove that the map is not zero it is enough to show that the constant term map $\phi \mapsto \phi_{Q}$ is not identically zero on $\pi$. But it is well known (e.g. JJac84]) that $\phi \mapsto \phi_{P}$ is not identically zero (in fact $\phi \mapsto \phi_{P}$ defines an imbedding of $L(\sigma, 2 m+1)$ in $\left.I\left(\tau,-\Lambda_{2 m+1}\right)\right)$ and we have

$$
\phi_{P}(g)=\int_{(U \cap L)(F) \backslash(U \cap L)(\mathbb{A})} \phi_{Q}(u g) d u .
$$

It therefore only remains to show that $\phi_{Q}[e]$ lies in the space of the automorphic representation $\sigma[-m] \otimes L(\sigma, 2 m)\left[\frac{1}{2}\right]$ of $L(\mathbb{A})$. To see this we use the automorphic realization of $L(\sigma, 2 m+1)$ and compute the constant term of multi-residues of Eisenstein series. Denote by $w_{Q}$ the longest element in ${ }_{L} W_{M}$. Thus, $w_{Q} \in{ }_{M} W_{M}^{c}$ and as a permutation in $S_{2 m+1}$ it is the cycle $(1,2, \ldots, 2 m+1)$. Let

$$
\mu_{Q}=w_{Q} \Lambda_{2 m+1}-\Lambda^{Q}=\left(-m, \frac{1}{2}\right) \in \mathfrak{a}_{L}^{*} .
$$


The formula for the constant term that we obtain in Lemma 1, implies that $E_{-1}(\varphi)_{Q}$ lies in the image of the operator

$$
E_{-1}^{Q}\left(\mu_{Q}\right): I\left(\tau, \Lambda_{2 m+1}\right) \rightarrow I_{Q}^{G}\left(\sigma \otimes L(\sigma, 2 m), \mu_{Q}\right)
$$

for $E_{-1}(\varphi)$ in the space of $\pi$ and therefore that $E_{-1}(\varphi)_{Q}[e]$ lies in $\sigma[-m] \otimes L(\sigma, 2 m)\left[\frac{1}{2}\right]$. It remains only to compute the constant term.

Lemma 1. For every $\varphi \in I(\tau)$ we have

$$
E_{-1}(\varphi)_{Q}=E_{-1}^{Q}\left(\left(M_{-1}\left(w_{Q}\right) \varphi\right), \mu_{Q}\right) .
$$

Proof. For $\varphi \in I(\tau)$ the constant term of the Eisenstein series $E(\varphi, \lambda)$ is given by

$$
E(\varphi, \lambda)_{Q}=\sum_{w \in \in_{L} W_{M}^{c}} E^{Q}(M(w, \lambda) \varphi, w \lambda)
$$

The multi-residue operator $\lim _{\lambda \rightarrow \Lambda_{2 m+1}} \prod_{i=1}^{2 m}\left(\lambda_{i}-\lambda_{i+1}-1\right)$ and the constant term operator are interchangeable. We show that after applying the multi-residue operator to (7), only the term associated with $w_{Q}$ survives. The map $w \mapsto w^{-1}(1)$ is a bijection from ${ }_{L} W_{M}^{c}$ to $[1,2 m+1]$. Let $w^{(i)} \in{ }_{L} W_{M}^{c}$ be such that $w^{(i)}(i)=1$, thus $w^{(i)}=(1,2, \ldots, i)$ (and in particular $w^{(2 m+1)}=w_{Q}$ ). For the term associated to the identity element $w^{(1)}$, note that $E^{Q}(\varphi, \lambda) \prod_{i=2}^{2 m}\left(\lambda_{i}-\lambda_{i+1}-1\right)$ is holomorphic at $\Lambda_{2 m+1}$. Therefore, the contribution to (77) of the term associated to the identity Weyl element vanishes after taking the multiresidue operator. For $i>1$ we have $\Delta\left(w^{(i)}\right)=\{i-1\}$ and therefore $\left(\lambda_{i-1}-\lambda_{i}-1\right) M\left(w^{(i)}, \lambda\right)$ is holomorphic at $\Lambda_{2 m+1}$. Note also that

$$
\begin{array}{r}
\left\{\left(w^{(i)}\right)^{-1}(j): j \in[1,2 m] \text { and }\left(w^{(i)} \Lambda_{2 m+1}\right)_{j}-\left(w^{(i)} \Lambda_{2 m+1}\right)_{j+1}=1\right\} \\
=[1,2 m] \backslash\{i-1, i\}
\end{array}
$$

and therefore that

$$
E^{Q}\left(M\left(w^{(i)}, \lambda\right) \varphi, w^{(i)} \lambda\right) \prod_{j \in[1,2 m] \backslash\{i\}}\left(\lambda_{j}-\lambda_{j+1}-1\right)
$$

is holomorphic at $\Lambda_{2 m+1}$. Thus if $i<2 m+1$ the $w^{(i)}$ contribution to (17) vanishes after applying the multi-residue operator. It follows that

$$
E_{-1}(\varphi)_{Q}=\lim _{\lambda \rightarrow \Lambda_{2 m+1}} E^{Q}\left(M\left(w_{Q}, \lambda\right) \varphi, w_{Q} \lambda\right) \prod_{i=1}^{2 m}\left(\lambda_{i}-\lambda_{i+1}-1\right) .
$$

The lemma follows.

This completes the proof of Proposition 1. 
As we already explained, this completes the proof of Theorem 4 . It follows from Lemma 1 that we can express the mixed period as

$$
l_{H}^{\psi_{r}}\left(E_{-1}(\varphi)\right)=\left(l_{U_{r}}^{\psi_{r}} \otimes l_{S p(2 m r)}\right)\left(E_{-1}^{Q}\left(M_{-1}\left(w_{Q} \varphi\right), \mu_{Q}\right)[e]\right)
$$

where $f[e] \in \sigma[-m] \otimes L(\sigma, 2 m)\left[\frac{1}{2}\right]$ is the valuation at $e$ of an element $f \in I_{Q}^{G}\left(\left(\sigma \otimes L(\sigma, 2 m), \mu_{Q}\right)\right.$.

Corollary 1. The mixed period integral $l_{H_{n-2 \kappa(\pi), 2 \kappa(\pi)}}^{\psi_{n-2 \kappa(\pi)}}$ is factorizable on the discrete spectrum representation $\pi$.

Proof. It is well known that on a cuspidal representation, the Whittaker functional is factorizable. It also follows from the explicit formula in Off06a, Theorem 1.1], that the purely symplectic period is factorizable on the residual spectrum. It then follows from (8) that the mixed period $l_{H_{r, 2 m r}}^{\psi_{r}}$ is factorizable on $L(\sigma, 2 m+1)$ for $\sigma$ a cuspidal representation of $G_{r}(\mathbb{A})$.

Remark 2. Note that the factorization of the period is obtained using a formula for the mixed period despite the fact that local multiplicity one for the mixed models is not yet known. Note also that based on the conjectural disjointness of models for unitary representations we expect the period $l_{H_{r, 2 k}}^{\psi}$ to vanish on every discrete spectrum representation $\pi$ such that $\kappa(\pi) \neq k$.

2.3. Some explicit formulas for the periods. Formula (8) indicates that the mixed period is related to special values of the RankinSelberg $L$-function associated to $\sigma$ and its contragradient $\tilde{\sigma}$. Let $L_{\sigma}(s)=$ $L(s, \sigma \times \tilde{\sigma})$ and fix once and for all a finite set of places $S$ containing the infinite places and such that for $v \notin S$ the conductor of $\psi_{v}$ is $\mathcal{O}_{v}$. If $\sigma$ is an everywhere unramified cuspidal representation of $G(\mathbb{A})$ and $\phi_{0}$ is its $L^{2}$-normalized spherical vector, it may be that $l_{U_{n}}^{\psi_{n}}\left(\phi_{0}\right)$ equals zero, but in any case we can write $l_{U_{n}}^{\psi_{n}}\left(\sigma(x) \phi_{0}\right)=\prod_{v} W_{v}^{\psi_{v}}(x)$ where $W_{v}^{\psi_{v}}$ is the spherical Whittaker function for all $v$, and it is normalized so that $W_{v}^{\psi_{v}}(e)=1$ for all $v \notin S$. Clearly, there exists $g \in G(\mathbb{A})$ such that $l_{U_{n}}^{\psi_{n}}\left(\sigma(g) \phi_{0}\right) \neq 0$ (in fact, we may and do choose $g$ such that $g_{v}=e$ for $v \notin S)$. We denote by $W_{1, v}^{\psi_{v}}$ the $L^{2}$-normalized spherical Whittaker function and refer to [LO07, §2.2] for the normalization of $W_{1, v}^{\psi_{v}}$ and for more details. It follows from Jacquet's formula for the inner product of cusp forms that

$$
\prod_{v \in S}\left|\frac{W_{v}^{\psi_{v}}(g)}{W_{1, v}^{\psi_{v}}(g)}\right|^{2}=\frac{1}{\operatorname{res}_{s=1} L_{\sigma}^{S}(s)}
$$


where $L_{\sigma}^{S}(s)$ is the partial $L$-function away from $S$. We then have

$$
\left|l_{U_{n}}^{\psi}\left(\pi(g) \phi_{0}\right)\right|^{2}=\frac{\prod_{v \in S} \alpha_{v}\left(\sigma_{v} ; g_{v}\right)}{\operatorname{res}_{s=1} L_{\sigma}(s)}
$$

where

$$
\alpha_{v}\left(\sigma_{v} ; g_{v}\right)=L_{\sigma_{v}}(1)\left|W_{1, v}^{\psi_{v}}\left(g_{v}\right)\right|^{2} .
$$

Assume now that $\sigma$ is an everywhere unramified cuspidal representation of $G_{r}(\mathbb{A})$ and let $\phi_{0}$ be the $L^{2}$-normalized, spherical element of the discrete spectrum representation $\pi=L(\sigma, t)$. We have the following formula for the mixed period of $\phi_{0}$.

Proposition 2. For a certain normalization of Haar measures independent of $\sigma$ we have that if $t=2 m$ is even then

$$
\left|l_{S p(n)} \phi_{0}\right|^{2}=\frac{L_{\sigma}(2) L_{\sigma}(4) \cdots L_{\sigma}(2 m)}{\operatorname{res}_{s=1} L_{\sigma}(s) L_{\sigma}(3) \cdots L_{\sigma}(2 m-1)}
$$

and if $t=2 m+1$ is odd then there exists an element $g_{0} \in G_{r}(\mathbb{A})$ such that

$$
\left|l_{H_{r, 2 m r}}^{\psi}\left(\pi\left(\operatorname{diag}\left(g_{0}, 1_{2 m r}\right)\right) \phi_{0}\right)\right|^{2}=\frac{\prod_{v \in S} \alpha_{v}\left(\sigma_{v} ; g_{0, v}\right)}{\operatorname{res}_{s=1} L_{\sigma}(s)} \prod_{j=1}^{m} \frac{L_{\sigma}(2 j)}{L_{\sigma}(2 j+1)} .
$$

Proof. When $t$ is even, the result is Off06b, Theorem 4] and when $t=1$ the formula is (91). Let $v_{0}$ be the $L^{2}$-normalized spherical cusp form in the space of $\sigma$ and let $\varphi_{0}^{(t)}$ be the spherical section in $I\left(\tau, \Lambda_{t}\right)$ normalized so that $\varphi_{0}^{(t)}(e)=v_{0}^{\otimes t}$. We now prove the formula for $t=2 m+1>1$ using (8) and a computation similar to that in Off06b. Indeed, we have

$$
\phi_{0}=\frac{E_{-1}\left(\varphi_{0}^{(t)}\right)}{\left\|E_{-1}\left(\varphi_{0}^{(t)}\right)\right\|_{2}} .
$$

As explained in [Off06b], Langlands showed that

$$
\left\|E_{-1}\left(\varphi_{0}^{(t)}\right)\right\|_{2}^{-2}=\frac{L_{\sigma}(2) L_{\sigma}(3) \cdots L_{\sigma}(t)}{\left(\operatorname{res}_{s=1} L_{\sigma}(s)\right)^{t-1}}
$$

(note the typo in the proof of [Off06b, Theorem 4] where $\left\|E_{-1}\left(\varphi_{0}\right)\right\|_{2}^{2}$ should be replaced by $\left.\left\|E_{-1}\left(\varphi_{0}\right)\right\|_{2}^{-2}\right)$. On the other hand

$$
M_{-1}\left(w_{Q}\right) \varphi_{0}^{(2 m+1)}=\frac{\operatorname{res}_{s=1} L_{\sigma}(s)}{L_{\sigma}(2 m+1)} \varphi_{0}^{(2 m+1)}
$$

and therefore

$$
E_{-1}^{Q}\left(M_{-1}\left(w_{Q}\right) \varphi_{0}^{(2 m+1)}, \mu_{Q}\right)[e]=\frac{\operatorname{res}_{s=1} L_{\sigma}(s)}{L_{\sigma}(2 m+1)}\left(v_{0} \otimes E_{-1}^{G_{2 m r}}\left(\varphi_{0}^{(2 m)}\right)\right) .
$$


The computation in the proof of [Off06b, Theorem 4] gives

$$
l_{S p(2 m r)}\left(E_{-1}^{G_{2 m r}}\left(\varphi_{0}^{(2 m)}\right)\right)=\frac{\left(\operatorname{res}_{s=1} L_{\sigma}(s)\right)^{m-1}}{L_{\sigma}(3) L_{\sigma}(5) \cdots L_{\sigma}(2 m-1)} .
$$

Plugging all this to (8) we get that

$$
\left|l_{H_{r, 2 m r}}^{\psi_{r}}\left(\phi_{0}\right)\right|^{2}=\left|l_{U_{r}}^{\psi_{r}}\left(v_{0}\right)\right|^{2} \frac{L_{\sigma}(2) L_{\sigma}(4) \cdots L_{\sigma}(2 m)}{L_{\sigma}(3) L_{\sigma}(5) \cdots L_{\sigma}(2 m+1)} .
$$

As already explained, for some everywhere unramified cuspidal representations $\sigma$ it may be that $l_{U_{r}}^{\psi_{r}}\left(v_{0}\right)=0$, however, there exists $g_{0} \in$ $G_{r}(\mathbb{A})$ such that $l_{U_{r}}^{\psi_{r}}\left(\sigma\left(g_{0}\right) v_{0}\right) \neq 0$. A similar computation then gives that for $g=\operatorname{diag}\left(g_{0}, 1_{2 m r}\right)$ we have

$$
\left|l_{H_{r, 2 m r}}^{\psi_{r}}\left(\pi(g) \phi_{0}\right)\right|^{2}=\left|l_{U_{r}}^{\psi_{r}}\left(\sigma\left(g_{0}\right) v_{0}\right)\right|^{2} \frac{L_{\sigma}(2) L_{\sigma}(4) \cdots L_{\sigma}(2 m)}{L_{\sigma}(3) L_{\sigma}(5) \cdots L_{\sigma}(2 m+1)} .
$$

The formula now follows from (9)).

\section{LOCAL MIXED MODELS}

Let $F$ be a non-archimedean local field of characteristic zero. For any algebraic group $Y$ defined over $F$ we denote from now on by $Y$ the group $Y(F)$ of $F$-rational points. Our goal is to prove Theorem 2 , i.e. to show that for any irreducible, unitary representation $\pi$ of $G$ we can attach an integer $\kappa(\pi) \in\left[0,\left[\frac{n}{2}\right]\right]$ so that

$$
\operatorname{Hom}_{H_{n-2 \kappa(\pi), 2 \kappa(\pi)}}\left(\pi, \psi_{n-2 \kappa(\pi)}\right) \neq 0 \text {. }
$$

The index $\kappa(\pi)$ is expressed in terms of the classification of the unitary dual of $G$ obtained by Tadic in Tad86]. Our proof is based on the properties of derivatives for representations of $G$ introduced by Gelfand-Kazhdan in GK75]. In [Zel80, Theorem 6.1], Zelevinsky classified all irreducible representation of $G$ in terms of cuspidal representations. Furthermore, the highest derivative of any irreducible representation is irreducible. The derivatives are computed by Zelevinsky in [Zel80, Theorem 8.1]. This computation is for derivatives in the 'opposite direction' to those suited for the study of Klyachko models with respect to the pairs $\left(H_{r, 2 k}, \psi_{r}\right)$. It will be more convenient to apply Zelevinsky's results as they stand. We therefore begin by introducing a closely related family of mixed models compatible with the derivatives computed by Zelevinsky. 
3.1. Another family of mixed models. The derivatives computed by Zelevinsky are more suited to the study of mixed models with respect to the pairs $\left(H_{2 k, r}^{\prime}, \psi_{r}^{\prime}\right)$ where

$$
H_{2 k, r}^{\prime}=\left\{\left(\begin{array}{cc}
h & X \\
0 & u
\end{array}\right): h \in S p(2 k), u \in U_{r}, X \in M_{2 k \times r}\right\}
$$

and

$$
\psi_{r}^{\prime}\left(\begin{array}{cc}
h & X \\
0 & u
\end{array}\right)=\psi\left(u_{1,2}+\cdots+u_{r-1, r}\right)
$$

whenever $u=\left(u_{i, j}\right) \in U_{r}$. The next lemma is relating between the two families of mixed models.

Lemma 2. Let $(\pi, V)$ be a representation of $G$. There is a linear isomorphism

$$
\operatorname{Hom}_{H_{r, 2 k}}\left(\pi, \psi_{r}\right) \simeq \operatorname{Hom}_{H_{2 k, r}^{\prime}}\left(\tilde{\pi}, \bar{\psi}_{r}^{\prime}\right) .
$$

Proof. Fix $r$ and $2 k$ and let $H=H_{r, 2 k}$ and $H^{\prime}=H_{2 k, r}^{\prime}$. Let $\tau$ be the involution on $G$ defined by

$$
\tau(g)=w^{t} g^{-1} w^{-1}
$$

where

$$
w=\left(\begin{array}{cc}
0 & w_{r} \\
1_{2 k} & 0
\end{array}\right)
$$

Let $\left(\pi^{\tau}, V\right)$ be the representation of $G$ on $V$ given by $\pi^{\tau}(g) v=\pi(\tau(g)) v$. It is well known ([GK75]) that $\pi^{\tau}$ is isomorphic to $\tilde{\pi}$ and therefore that there is a linear isomorphism

$$
\operatorname{Hom}_{H^{\prime}}\left(\tilde{\pi}, \bar{\psi}_{r}^{\prime}\right)=\operatorname{Hom}_{H^{\prime}}\left(\pi^{\tau}, \bar{\psi}_{r}^{\prime}\right) .
$$

Note further that $\tau(H)=H^{\prime}$ and that $\psi_{r}(h)=\bar{\psi}_{r}^{\prime}(\tau(h))$. This implies that the identity map on the space of $\pi$ defines a linear isomorphism

$$
\operatorname{Hom}_{H}\left(\pi, \psi_{r}\right)=\operatorname{Hom}_{H^{\prime}}\left(\pi^{\tau}, \bar{\psi}_{r}^{\prime}\right) .
$$

3.2. The dual of $G$. Zelevinski classified in [Zel80] all irreducible representations of $G$ in terms of cuspidal representations. For a representation $\sigma$ of $G_{r}$ and for $\lambda \in \mathbb{C}$ let $\sigma[\lambda]=|\operatorname{det}|^{\lambda} \sigma$. If $\sigma_{i}$ is a representation of $G_{r_{i}}, i=1, \ldots, t$ we denote by $\sigma_{1} \times \cdots \times \sigma_{t}$ the representation of $G_{r_{1}+\cdots+r_{t}}$ parabolically induced from $\sigma_{1} \otimes \cdots \otimes \sigma_{t}$. Let $\mathcal{C}$ denote the collection of all irreducible, cuspidal representations of $G_{r}$ for all positive integers $r$. For any $a, b \in \mathbb{R}$ such that $0 \leq b-a \in \mathbb{Z}$ and any $\rho \in \mathcal{C}$ the set

$$
\Delta=[a, b]^{(\rho)}=\{\rho[a+i]: 0 \leq i \leq b-a\}
$$


is called a segment. The representation $\rho[a] \times \rho[a+1] \times \cdots \times \rho[b]$ has a unique irreducible subrepresentation which is denoted by $\langle\Delta\rangle$. We say that a segment $\Delta=[a, b]^{(\rho)}$ precedes the segment $\Delta^{\prime}=\left[a^{\prime}, b^{\prime}\right]^{(\rho)}$ if $\Delta^{\prime} \not \subset \Delta, \rho^{\prime}\left[a^{\prime}\right]=\rho[a+k]$ for some integer $k>0$ and $\Delta \cup \Delta^{\prime}$ is also a segment. Denote by $\mathcal{O}$ the collection of all multisets of segments $[a, b]^{(\rho)}$ with $\rho \in \mathcal{C}$ and $b-a$ a non negative integer. For any $a \in \mathcal{O}$ the segments in $a$ can be arranged as $a=\left\{\Delta_{1}, \ldots, \Delta_{t}\right\}$ so that for all $1 \leq i<j \leq t$ the segment $\Delta_{i}$ does not precede $\Delta_{j}$. In this case the representation $\left\langle\Delta_{1}\right\rangle \times \cdots \times\left\langle\Delta_{t}\right\rangle$ has a unique irreducible subrepresentation denoted by $\langle a\rangle$. This is the statement of [Zel80, Theorem 6.1 (a)]. The rest of [Zel80, Theorem 6.1] is the following statement.

Theorem 5 (Zelevinsky). Any irreducible representation of $G$ is of the form $\langle a\rangle$ for some $a \in \mathcal{O}$ uniquely determined by the multiset $a$.

3.3. Derivatives of representations of $G$. Derivatives of representations of $G$ were introduced in GK75]. For a non negative integer $k \leq n$ the $k$ th derivative is a functor taking a representation $\pi$ of $G$ to a representation $\pi^{(k)}$ of $G_{n-k}$. It is defined as follows. Let $P_{k}$ be the mirabolic subgroup of matrices in $G_{k}$ with last row $(0, \ldots, 0,1)$ and let $V_{k}$ be its unipotent radical. We imbed $G_{k-1}$ in the upper left block of $G_{k}$ whenever convenient. The functors $\Phi^{-}$from representations of $P_{k}$ to representations of $P_{k-1}$ and $\Psi^{-}$from representations of $P_{k}$ to representations of $G_{k-1}$ are defined in [BZ77, §3.2]. For a representation $\tau$ of $P_{k}, \Phi^{-}(\tau)$ is the normalized Jacquet functor of $\tau$ with respect to $V_{k}$ and the character $\theta(v)=\psi\left(v_{k-1, k}\right), v=\left(v_{i, j}\right) \in V_{k}$ regarded as a representation of $P_{k-1}$ imbedded in $P_{k}$ and $\Psi^{-}(\tau)$ is the normalized Jacquet module of $\tau$ with respect to $V_{k}$ and the trivial character regarded as a representation of $G_{k-1}$ imbedded in $P_{k}$. If $\Phi^{-(m)}$ denotes the functor $\Phi^{-}$applied $m$ times (hence it is a functor from representations of $P_{n}$ to representations of $P_{n-m}$ ) and $\pi$ is a representation of $G$ then the $r$ th derivative of $\pi$ is given by

$$
\pi^{(r)}=\Psi^{-} \Phi^{-(r)}\left(\pi_{\mid P_{n}}\right) .
$$

The main property of derivatives relevant to the study of mixed models lies in the content of [Zel80, Proposition 3.7]. We now recall this property. For any representation $(\pi, V)$ of $G_{n}$ let $\left(\pi^{(r)}, V^{(r)}\right)$ be the $r$ th derivative. Then there is a surjective morphism $A=A_{\psi}^{(r)}(\pi): V \rightarrow V^{(r)}$ so that

$$
A\left(\pi\left(\begin{array}{cc}
g & X \\
0 & u
\end{array}\right) v\right)=\psi_{r}(u) \pi^{(r)}(g)(A v)
$$


for all $g \in G_{n-r}, u \in U_{r}, X \in M_{n-r \times r}$ and $v \in V$. For any subgroup $Y$ of $G_{n-r}$ let

$$
H_{Y, r}=\left\{\left(\begin{array}{cc}
y & X \\
0 & u
\end{array}\right): y \in Y, X \in M_{n-r \times r}, u \in U_{r}\right\} .
$$

the map $A$ provides the identification

$$
\operatorname{Hom}_{H_{Y, r}}\left(\pi, \psi_{r}\right)=\operatorname{Hom}_{Y}\left(\pi^{(r)}, 1\right)
$$

The functor of $m$ th derivative satisfies a 'Leibnitz rule'. In BZ77, Lemma 4.5], it is proved that for representations $\sigma_{1}$ of $G_{k}$ and $\sigma_{2}$ of $G_{r}$ the representation $\left(\sigma_{1} \times \sigma_{2}\right)^{(m)}$ is glued together from $\sigma_{1}^{(i)} \times \sigma_{2}^{(m-i)}, i=$ $0, \ldots, m$, i.e. that there is a filtration on $\left(\sigma_{1} \times \sigma_{2}\right)^{(m)}$ with factors $\sigma^{(i)} \times \sigma_{2}^{(m-i)}$. An easy consequence of [BZ77, Proposition 4.13 (a),(b)] is the following.

Lemma 3. There exists a surjective morphism from $\left(\sigma_{1} \times \sigma_{2}\right)^{(m)}$ to $\sigma_{1} \times \sigma_{2}^{(m)}$.

Proof. It follows from [BZ77, Proposition 4.13 (a)] that there is a surjective morphism

$$
\left(\sigma_{1} \times \sigma_{2}\right)_{\mid P_{k+r}} \rightarrow \sigma_{1} \times\left(\sigma_{2 \mid P_{r}}\right)
$$

(see [BZ77, p. 457-458] for the meaning of the induced representation $\left.\sigma_{1} \times\left(\sigma_{2 \mid P_{r}}\right)\right)$. Let $\Omega$ be either $\Phi^{-}$or $\Psi^{-}$. From [BZ77, Proposition 4.13 (b)] for a representation $\tau$ of $P_{r}$ we have

$$
\Omega\left(\sigma_{1} \times \tau\right)=\sigma_{1} \times \Omega(\tau) .
$$

Together with the exactness of $\Omega$ this implies that there is a surjective morphism

$$
\Omega\left(\left(\sigma_{1} \times \sigma_{2}\right)_{\mid P_{k+r}}\right) \rightarrow \sigma_{1} \times \Omega\left(\sigma_{2 \mid P_{r}}\right) .
$$

Iterating this argument the lemma follows from the definition of the derivative.

If $\pi^{(k)} \neq 0$ and $\pi^{(m)}=0$ for all $m>k$ then $\pi^{(k)}$ is called the highest derivative of $\pi$. For any segment $\Delta=[a, b]^{(\rho)}$ let $\Delta^{-}=[a, b-1]^{(\rho)}$. If $a \in \mathcal{O}$ is any multiset of segments, let $a^{-}$be the multiset of segments $\Delta^{-}$so that $\Delta$ is a segment in $a$ and $\Delta^{-}$is not empty.

Theorem 6 ([Zel80], Theorem 8.1). For all $a \in \mathcal{O}$ the highest derivative of $\langle a\rangle$ is $\left\langle a^{-}\right\rangle$. 
3.4. The unitary dual of $G$. We briefly review the classification of Tadic for the unitary dual of the general linear groups ([Tad86, Theorem D]). A representation $\delta$ of $G_{r}$ is called square integrable if its matrix coefficients belong to $L^{2}\left(Z_{G} \backslash G\right)$. Denote by $D^{u}$ the collection of all irreducible, square integrable representations of $G_{r}$ with $r$ ranging over all positive integers. For $\delta \in D^{u}$ and a positive integer $t$ the representation

$$
\delta\left[\frac{1-t}{2}\right] \times \delta\left[\frac{3-t}{2}\right] \times \cdots \times \delta\left[\frac{t-1}{2}\right]
$$

has a unique irreducible subrepresentation which we denote by $U(\delta, t)$. It will be convenient to allow the notation $U(\delta, 0)$ for the trivial representation of $G_{0}=\{e\}$. Let $B$ be the collection of all representations of the form $U(\delta, t)$ or $U(\delta, t)[\alpha] \times U(\delta, t)[-\alpha]$ where $\delta \in D^{u}, t$ is a positive integer and $0<\alpha<\frac{1}{2}$.

Theorem 7 (Tadic). For every $\sigma_{1}, \ldots, \sigma_{t} \in B$ the representation $\sigma_{1} \times \cdots \times \sigma_{t}$ is irreducible and unitary. Any irreducible, unitary representation of $G$ is of this form uniquely determined (up to reordering) by the multiset $\left\{\sigma_{1}, \ldots, \sigma_{t}\right\}$.

3.5. Derivatives of Speh representations. We will have to compute highest derivatives for certain representations induced from Speh representations. In order to be able to apply Theorem 6, we need to express the representations of the form $U(\delta, t)[\alpha]$ in terms of the classification of Zelevinsky for the dual of $G$, i.e. we wish to describe explicitly the set $a=a(\delta, t, \alpha) \in \mathcal{O}$ such that $U(\delta, t)[\alpha]=\langle a\rangle$. For any $\delta \in D^{u}$ there is an irreducible, cuspidal, unitary representation $\rho \in \mathcal{C}$ and a positive integer $d$ such that $\delta$ is the unique irreducible subrepresentation of

$$
\rho\left[\frac{d-1}{2}\right] \times \cdots \times \rho\left[\frac{1-d}{2}\right],
$$

i.e. such that $\delta=\left\langle\left\{\rho\left[\frac{d-1}{2}\right], \rho\left[\frac{d-3}{2}\right], \ldots, \rho\left[\frac{1-d}{2}\right]\right\}\right\rangle$ is given in terms of singleton segments. Let

$$
\Delta(t, \rho)=\left[\frac{1-t}{2}, \frac{t-1}{2}\right]^{(\rho)} .
$$

In Tad86, Theorem A. 10 (iii)] it is proved that

$$
U(\delta, t)=\left\langle\left\{\Delta\left(t, \rho\left[\frac{1-d}{2}\right]\right), \Delta\left(t, \rho\left[\frac{3-d}{2}\right]\right), \ldots, \Delta\left(t, \rho\left[\frac{d-1}{2}\right]\right)\right\}\right\rangle .
$$

As pointed out in [Tad95, Theorem 3.2], for any $\alpha \in \mathbb{R}$ we then have $a(\delta, t, \alpha)=\left\{\Delta\left(t, \rho\left[\frac{1-d}{2}+\alpha\right]\right), \Delta\left(t, \rho\left[\frac{3-d}{2}+\alpha\right]\right), \ldots, \Delta\left(t, \rho\left[\frac{d-1}{2}+\alpha\right]\right)\right\}$. 
Note that

$$
\Delta(t, \rho)^{-}=\Delta\left(t-1, \rho\left[-\frac{1}{2}\right]\right)
$$

and therefore that

$a(\delta, t, \alpha)^{-}=\left\{\Delta\left(t-1, \rho\left[\frac{1-d}{2}+\left(\alpha-\frac{1}{2}\right)\right]\right), \ldots, \Delta\left(t-1, \rho\left[\frac{d-1}{2}+\left(\alpha-\frac{1}{2}\right)\right]\right)\right\}$.

By Theorem 6 we get that the highest derivative of $U(\delta, t)[\alpha]$ is

$$
U(\delta, t-1)\left[\alpha-\frac{1}{2}\right]
$$

See [SS90, Theorem 3] for an analog for $G L_{n}(\mathbb{R})$. Applying the Leibnitz rule for derivatives and an easy inductive argument we obtain the following.

Lemma 4. Let $\delta, \ldots, \delta_{m} \in D^{u}, t_{1}, \ldots, t_{m}$ positive integers and $\alpha_{1}, \ldots, \alpha_{m} \in$ $\mathbb{R}$. The highest derivative of the representation

$$
U\left(\delta_{1}, t_{1}\right)\left[\alpha_{1}\right] \times \cdots \times U\left(\delta_{m}, t_{m}\right)\left[\alpha_{m}\right]
$$

is the representation

$$
U\left(\delta_{1}, t_{1}-1\right)\left[\alpha_{1}-\frac{1}{2}\right] \times \cdots \times U\left(\delta_{m}, t_{m}-1\right)\left[\alpha_{m}-\frac{1}{2}\right] .
$$

Proof. Let $\delta_{i}$ be a representation of $G_{r_{i}}$. We wish to show that

$$
\begin{aligned}
& \left(U\left(\delta_{1}, t_{1}\right)\left[\alpha_{1}\right] \times \cdots \times U\left(\delta_{m}, t_{m}\right)\left[\alpha_{m}\right]\right)^{(k)}= \\
& \begin{cases}0 & k>r_{1}+\cdots+r_{m} \\
U\left(\delta_{1}, t_{1}-1\right)\left[\alpha_{1}-\frac{1}{2}\right] \times \cdots \times U\left(\delta_{m}, t_{m}-1\right)\left[\alpha_{m}-\frac{1}{2}\right] & k=r_{1}+\cdots+r_{m} .\end{cases}
\end{aligned}
$$

We have already proved this when $m=1$. Assume by induction that this is true for $m-1$. By Leibnitz rule, the representation $\left(U\left(\delta_{1}, t_{1}\right)\left[\alpha_{1}\right] \times \cdots \times U\left(\delta_{m}, t_{m}\right)\left[\alpha_{m}\right]\right)^{(k)}$ is glued together from

$$
\left(U\left(\delta_{1}, t_{1}\right)\left[\alpha_{1}\right]\right)^{(i)} \times\left(U\left(\delta_{2}, t_{2}\right)\left[\alpha_{2}\right] \cdots \times U\left(\delta_{m}, t_{m}\right)\left[\alpha_{m}\right]\right)^{(k-i)}
$$

for $i=0, \ldots, k$. But if $k>r_{1}+\cdots+r_{m}$ then for each $i$ we have either $i>r_{1}$ or $k-i>r_{2}+\cdots+r_{m}$ and therefore it follows from the induction hypothesis that $\left(U\left(\delta_{1}, t_{1}\right)\left[\alpha_{1}\right] \times \cdots \times U\left(\delta_{m}, t_{m}\right)\left[\alpha_{m}\right]\right)^{(k)}=0$. Similarly, if $k=r_{1}+\cdots+r_{m}$ then any component with $i \neq r_{1}$ must vanish. It follows that

$$
\begin{aligned}
& \left(U\left(\delta_{1}, t_{1}\right)\left[\alpha_{1}\right] \times \cdots \times U\left(\delta_{m}, t_{m}\right)\left[\alpha_{m}\right]\right)^{\left(r_{1}+\cdots+r_{m}\right)}= \\
& \quad\left(U\left(\delta_{1}, t_{1}\right)\left[\alpha_{1}\right]\right)^{\left(r_{1}\right)} \times\left(U\left(\delta_{2}, t_{2}\right)\left[\alpha_{2}\right] \cdots \times U\left(\delta_{m}, t_{m}\right)\left[\alpha_{m}\right]\right)^{\left(r_{2}+\cdots+r_{m}\right)}
\end{aligned}
$$

and the lemma follows by the induction hypothesis. 
3.6. Klyachko Models for some representations of $G$. We are now ready to state our main local result.

Theorem 8. Let $\delta_{1}, \ldots, \delta_{q}, \delta_{1}^{\prime}, \ldots, \delta_{q^{\prime}}^{\prime} \in D^{u}$, let $m_{1}, \ldots, m_{q}, m_{1}^{\prime}, \ldots, m_{q^{\prime}}^{\prime}$ be non negative integers and let $\alpha_{1}, \ldots, \alpha_{q}, \alpha_{1}^{\prime}, \ldots, \alpha_{q^{\prime}}^{\prime} \in \mathbb{R}$. Assume that $\delta_{i}$ is a representation of $G_{r_{i}}$, that $\delta_{i}^{\prime}$ is a representation of $G_{r_{i}^{\prime}}$ and that

$$
n=\sum_{i=1}^{q}\left(2 m_{i}+1\right) r_{i}+\sum_{i=1}^{q^{\prime}} 2 m_{i}^{\prime} r_{i}^{\prime} .
$$

Let $n=r+2 k$ where

$$
r=r_{1}+\cdots+r_{q} \text { and } k=m_{1} r_{1}+\cdots+m_{q} r_{q}+m_{1}^{\prime} r_{1}^{\prime}+\cdots+m_{q^{\prime}} r_{q^{\prime}} .
$$

The representation

$$
\begin{aligned}
U\left(\delta_{1}^{\prime}, 2 m_{1}^{\prime}\right)\left[\alpha_{1}^{\prime}\right] & \times \cdots \times U\left(\delta_{q^{\prime}}^{\prime}, 2 m_{q^{\prime}}^{\prime}\right)\left[\alpha_{q^{\prime}}^{\prime}\right] \\
& \times U\left(\delta_{1}, 2 m_{1}+1\right)\left[\alpha_{1}\right] \times \cdots \times U\left(\delta_{q}, 2 m_{q}+1\right)\left[\alpha_{q}\right]
\end{aligned}
$$

is $\left(H_{r, 2 k}, \psi_{r}\right)$-distinguished.

Proof. For $\delta \in D^{u}$ the contragradiant of $U(\delta, t)$ is $U(\tilde{\delta}, t)$. Since the contragradiant of an induced representation $\sigma_{1} \times \cdots \times \sigma_{t}$ is $\tilde{\sigma}_{1} \times \cdots \times \tilde{\sigma}_{t}$ the contragradiant of a representation that has the form (13) is also of such a form. It therefore follows from Lemma 2 that the theorem is equivalent to the statement that representations $\pi$ of the form (13) are $\left(H_{2 k, r}^{\prime}, \psi_{r}^{\prime}\right)$-distinguished $(\psi$ and therefore $\bar{\psi}$ is an arbitrary non trivial character of $F$ ). Let

$$
\begin{gathered}
\sigma_{1}=U\left(\delta_{1}^{\prime}, 2 m_{1}^{\prime}\right)\left[\alpha_{1}^{\prime}\right] \times \cdots \times U\left(\delta_{q^{\prime}}^{\prime}, 2 m_{q^{\prime}}^{\prime}\right)\left[\alpha_{q^{\prime}}^{\prime}\right] \\
\sigma_{2}=U\left(\delta_{1}, 2 m_{1}+1\right)\left[\alpha_{1}\right] \times \cdots \times U\left(\delta_{q}, 2 m_{q}+1\right)\left[\alpha_{q}\right]
\end{gathered}
$$

and $\pi=\sigma_{1} \times \sigma_{2}$. By Lemma 3 there is a surjective morphism $\mathfrak{p}: \pi^{(r)} \rightarrow$ $\sigma_{1} \times \sigma_{2}^{(r)}$. There is also a surjective linear map $A: \pi \rightarrow \pi^{(r)}$ satisfying the equivariance properties of (10). By Lemma 4 we see that

$$
\begin{aligned}
\sigma_{1} \times \sigma_{2}^{(r)}=U\left(\delta_{1}^{\prime}, 2 m_{1}^{\prime}\right)\left[\alpha_{1}^{\prime}\right] \times \cdots \times U\left(\delta_{q^{\prime}}^{\prime}, 2 m_{q^{\prime}}^{\prime}\right)\left[\alpha_{q^{\prime}}^{\prime}\right] \times \\
U\left(\delta_{1}, 2 m_{1}\right)\left[\alpha_{1}-\frac{1}{2}\right] \times \cdots \times U\left(\delta_{q}, 2 m_{q}\right)\left[\alpha_{q}-\frac{1}{2}\right]
\end{aligned}
$$

is induced from Speh representations of the form $U(\delta, t)[\alpha]$ with $t$ even. By [OS07, Proposition 2], there exists a non zero element $\ell \in$ $\operatorname{Hom}_{S p(2 k)}\left(\sigma_{1} \times \sigma_{2}^{(r)}, \mathbb{C}\right)$. It follows that $\ell \circ \mathfrak{p} \circ A$ is a non zero element of $\operatorname{Hom}_{H_{2 k, r}^{\prime}}\left(\pi, \psi_{r}^{\prime}\right)$. 
Using the notation of the statement of Theorem 8 for a representation $\pi$ of the form (13) we define

$$
\kappa(\pi)=k .
$$

Note that by Theorem 7 every irreducible, unitary representation $\pi$ of $G$ is of the form (13) with $\left|\alpha_{i}\right|,\left|\alpha_{i}^{\prime}\right|<\frac{1}{2}$. In particular $\kappa(\pi)$ is defined. The following corollary is then immediate from Theorem 8 .

Corollary 2. Let $\pi$ be an irreducible, unitary representation of $G$ then $\pi$ is $\left(H_{n-2 \kappa(\pi), 2 \kappa(\pi)}, \psi_{n-2 \kappa(\pi)}\right)$-distinguished.

This proves in particular Theorem 2 .

\section{REFERENCES}

[BZ77] I. N. Bernstein and A. V. Zelevinsky. Induced representations of reductive §-adic groups. I. Ann. Sci. École Norm. Sup. (4), 10(4):441-472, 1977.

[FG03] Jason Fulman and Robert Guralnick. Conjugacy class properties of the extension of $G L(n, q)$ generated by the inverse tranpose involution, arxiv:math.gr/0304047 v1 3, 2003.

[GK75] I. M. Gelfand and D. A. Kajdan. Representations of the group GL $(n, K)$ where $K$ is a local field. In Lie groups and their representations (Proc. Summer School, Bolyai János Math. Soc., Budapest, 1971), pages 95-118. Halsted, New York, 1975.

[Heu93] Michael J. Heumos. Models and periods for automorphic forms on $\mathrm{GL}_{n}$. In Representation theory of groups and algebras, volume 145 of Contemp. Math., pages 135-144. Amer. Math. Soc., Providence, RI, 1993.

[HR90] Michael J. Heumos and Stephen Rallis. Symplectic-Whittaker models for $\mathrm{gl}_{n}$. Pacific J. Math., 146(2):247-279, 1990.

[Jac84] Hervé Jacquet. On the residual spectrum of $G L(n)$. In Lie group representations, II (College Park, Md., 1982/1983), pages 185-208. Springer, Berlin, 1984.

[JPSS83] H. Jacquet, I. I. Piatetskii-Shapiro, and J. A. Shalika. Rankin-Selberg convolutions. Amer. J. Math., 105(2):367-464, 1983.

[JR92] Hervé Jacquet and Stephen Rallis. Symplectic periods. J. Reine Angew. Math., 423:175-197, 1992.

[IS91] N. F. J. Inglis and J. Saxl. An explicit model for the complex representations of the finite general linear groups. Arch. Math. (Basel), 57(5):424431, 1991.

[K184] A. A. Klyachko. Models for complex representations of the groups GL $(n, q)$. Matematicheskiu Sbornik. Novaya Seriya, 48(2):365-378, 1984.

[LO07] Erez Lapid and Omer Offen. Compact unitary periods. Compositio Math., 143(2):323-338, 2007.

[MW89] C. Mœglin and J.-L. Waldspurger. Le spectre résiduel de GL(n). Ann. Sci. École Norm. Sup. (4), 22(4):605-674, 1989.

[N07] C. Nien. Klyachko Models for General Linear Groups of Rank 5 over a p-adic Field. Candian Journal of Mathematics, to appear.

[Off06a] Omer Offen. On symplectic periods of discrete spectrum of $G L_{2 n}$. Isr. Jour. Math., 154:253-298, 2006. 
[Off06b] Omer Offen. Distinguished residual spectrum. Duke Math. J., 134(2):313$357,2006$.

[OS07] Omer Offen and Eitan Sayag. On unitary representations of $G L_{2 n}$ distinguished by the symplectic group. J. Number Theory, 125:344-355, 2007.

[Rod73] François Rodier. Whittaker models for admissible representations of reductive $p$-adic split groups. In Harmonic analysis on homogeneous spaces (Proc. Sympos. Pure Math., Vol. XXVI, Williams Coll., Williamstown, Mass., 1972), pages 425-430. Amer. Math. Soc., Providence, R.I., 1973.

[SS90] Siddhartha Sahi and Elias M. Stein. Analysis in matrix space and Speh's representations. Invent. Math., 101(2):379-393, 1990.

[Tad86] Marko Tadić. Classification of unitary representations in irreducible representations of general linear group (non-Archimedean case). Ann. Sci. École Norm. Sup. (4), 19(3):335-382, 1986.

[Tad95] M. Tadić. On characters of irreducible unitary representations of general linear groups. Abh. Math. Sem. Univ. Hamburg, 65:341-363, 1995.

[TV05] Nathaniel Thiem and C. Ryan Vinroot. On the characteristic map of finite unitary groups, arxiv:math.rt/0511220 v1, 2005.

[Vin06] C. Ryan Vinroot. Involutions acting on representations. J. Algebra, 297(1):50-61, 2006.

[Zel80] A. V. Zelevinsky. Induced representations of reductive $p$-adic groups. II. On irreducible representations of GL(n). Ann. Sci. École Norm. Sup. (4), 13(2):165-210, 1980.

Humboldt-Universität Zu Berlin, Mathematich-Naturwissenschaftliche

Fakultät II, Institut für Mathematik, Sitz: Rudower Chausee 25, D10099 Berlin, Germany.

E-mail address: offen@mathematik.hu-berlin.de

Einstein Institute of Mathematics, Edmond J. Safra Campus, Givat

Ram, The Hebrew University of Jerusalem, Jerusalem, 91904, Israel

E-mail address: sayag@math.huji.ac.il 